\title{
Medical Image Fusion based on DWT and SPIHT Techniques with Quantitative Analysis
}

\author{
Gopal Kumar \\ M.Tech Scholar, \\ RKDFIST Bhopal (RGPV), India;
}

\author{
Manish Trivedi \\ HOD, RKDFIST \\ Bhopal (RGPV), India
}

\begin{abstract}
Medical image fusion has revolutionized medical analysis by raising the preciseness and performance of computer assisted diagnosing. This fused image is a lot of productive as compared to its original input images. The fusion technique in medical images is beneficial for resourceful disease diagnosing purpose. This paper illustrates completely different multimodality medical picture combination method and their consequences evaluate with various quantitative metrics. Firstly 2 registered pictures CT (anatomical information) and MRI-T2 (functional information) are taken as input. Then the fusion techniques are applied onto the input pictures such as Mamdani kind minimum-sum-mean of maximum (MIN-SUM-MOM) and Redundancy discrete wave transform (RDWT) and so the resulting fused image is analyzed with quantitative metrics namely Over all irritated Entropy, Peak Signal -to- Noise ratio (PSNR), Signal to Noise ratio (SNR), Structural Similarity Index(SSIM), Mutual Information(MI). From the derived results it's inferred that Mamdani type MIN-SUM-MOM is more productive than RDWT and also the projected fusion techniques provide additional info compared to the input images as justified by all the metrics.
\end{abstract}

\section{Keywords}

Signal processing method, precise estimation of , roughly observed data

\section{INTRODUCTION}

Image compression is minimizing the mass in bytes of a graphics file without demeaning the quality of the image to an unacceptable level. The reduction in file size permits additional images to be deposited in a given amount of disk or memory space. It also minimizes the time demanded for images to be transfer over the Internet or downloaded through Web pages.

There are several numerous techniques in which image files can be compressed. For Internet utilization, the two most general compressed graphic image arrangements are the JPEG scheme and the GIF scheme. The JPEG procedure is more often utilized for photographs, while the GIF procedure is generally utilized for line art and next images in which geometric shapes are relatively normal. Other methods for image compression involve the utilization of fractals and wavelets. These procedures have not gained widespread acceptance for utilization on the Internet as of this writing. However, both procedures give promise because they produce higher compression ratios as comparison to that of the JPEG or GIF procedures for some types of images. Another latest procedure that may in time substitute the GIF arrangement is the PNG formulation.

Compressing an image is importantly different than the compressing raw binary data. Of course, general-purpose compression programs can be utilized to compress images, but the output is less as that of the optimal. This is because images have certain statistical characteristics, which can be exploited by encoders specifically generated for them. Additionally, some of the finer information in the image can be relinquish for the sake of depositing a additional bandwidth or storage space. This also means that lossy compression procedures can be utilized in this field.

A text file or program can be compacted without the introduction of errors, but only up to a certain limit. This is termed as lossless compression. Above this point, errors are to be produced. In text and program files, it is significant that compression be lossless as a single error can seriously destruct the meaning of a text file, or reason a program not to sprint. In image solidity, a small loss in quality is generally not considerable. There is no "critical point" up to which solidity works correctly, but over which it becomes notpossible. When there is some forbearance for defeat, the compression factor can be better than it can when there is no loss forbearance. Due to this reason, graphic images can be compressed. more than text files or programs.

\section{METHOD}

\subsection{Preprocessing Of Image Fusion}

Two images taken in several angles of scene generally cause distortion. Most of objects are identical however the shapes amendment slightly. At the start of fusing pictures, we've to create positive that every component at related pictures has the association between pictures so as to repair the problem of distortion; image registration will do that. 2 pictures having same scene will register along exploitation software system to attach many management points. when registration, resembling is finished to regulate every image that on the brink of fuse to a similar dimension. when resembling, every image are going to be of a similar size. many interpolation approaches is used, to resample the image; the rationale is that almost all approaches we have a tendency to use ar all pixelby-pixel amalgamate. pictures with a similar size are going to be straightforward for fusing method. when the re-sampling, fusion algorithmic program is applied. typically we've to transfer the image into completely different domain, typically haven't reckoning on the algorithmic program. Inverse transfer is important if image has been transferred into another domain. Fig.1 summarizes these steps referred to as, preprocessing of image fusion. 


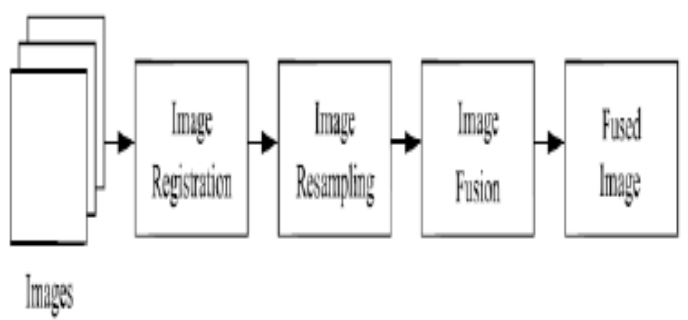

Fig.1. preprocessing of image fusion

Fusion of Multimodal Brain Images using RDWT

Medical images captured at totally different time instances can have variations because of geometric deformations. To optimally fuse $22 \mathrm{D} / 3 \mathrm{D}$ medical images, we tend to 1 st ought to minimize linear and non-linear variations between them using registration technique. Medical image registration is regarding deciding geometrical revolution that aligns points in one medical information set with corresponding points in another information set [6]. we tend to 1st propose mutual data based mostly non-linear registration algorithmic program for registering multimodal medical pictures. Mutual data could be a construct from scientific theory during which applied math dependence is measured between 2 random variables

\section{Image Compression Process}

A characteristic loss image compression system is shown in Fig. 1. primarily it involves 3 closely connected parts particularly (a) source Encoder (b) Quantizer, and (c) Entropy Encoder. Compression is expert by applying a linear remodel to decor relate the image information, quantizing the ensuing rework coefficients, and entropy coding the measure values.

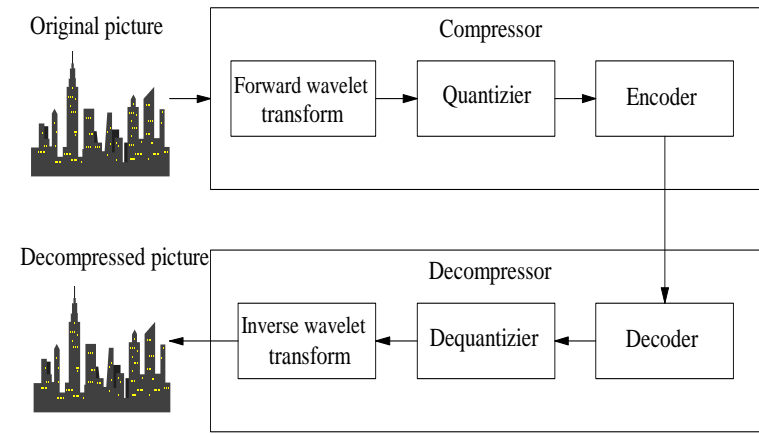

Fig.2. Image Compression Process

\section{RESULT}

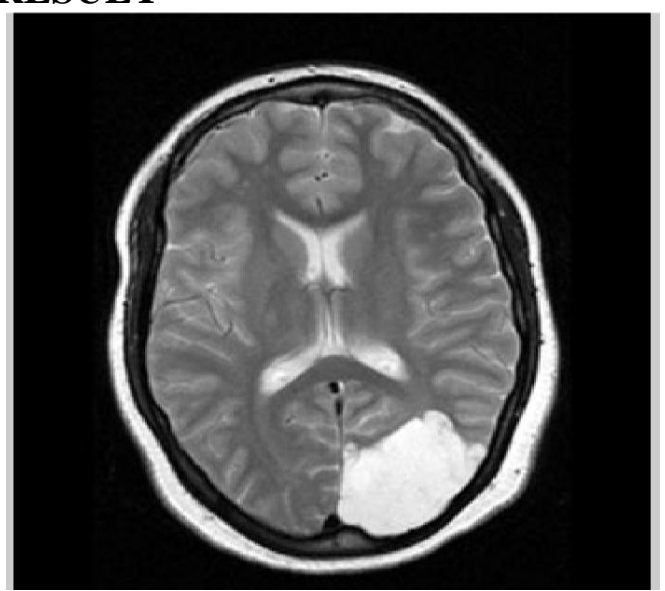

Fig .1. Original MRI Image

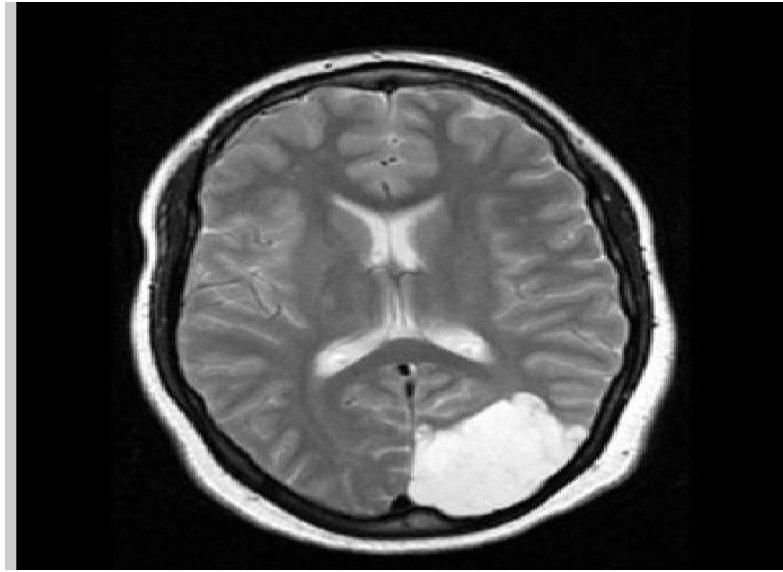

Fig.2.Gray Image

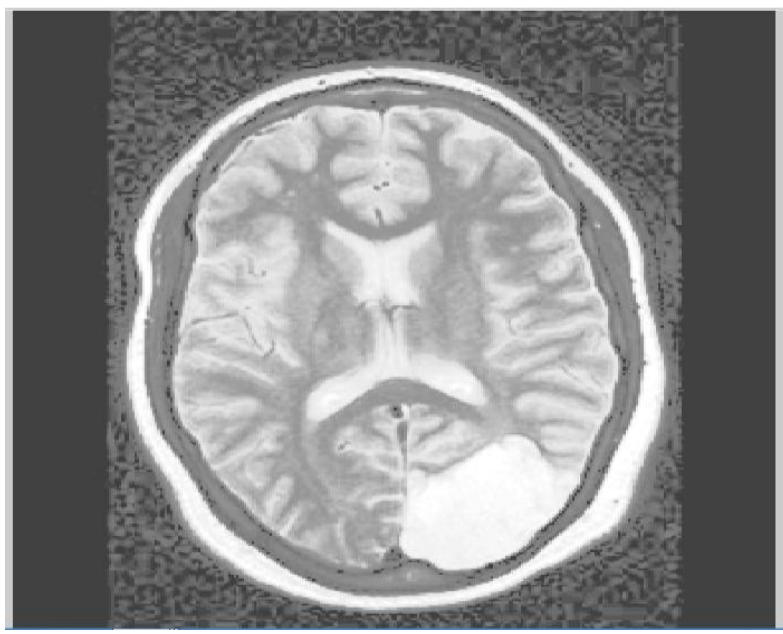

Fig.3. Enhanced Image
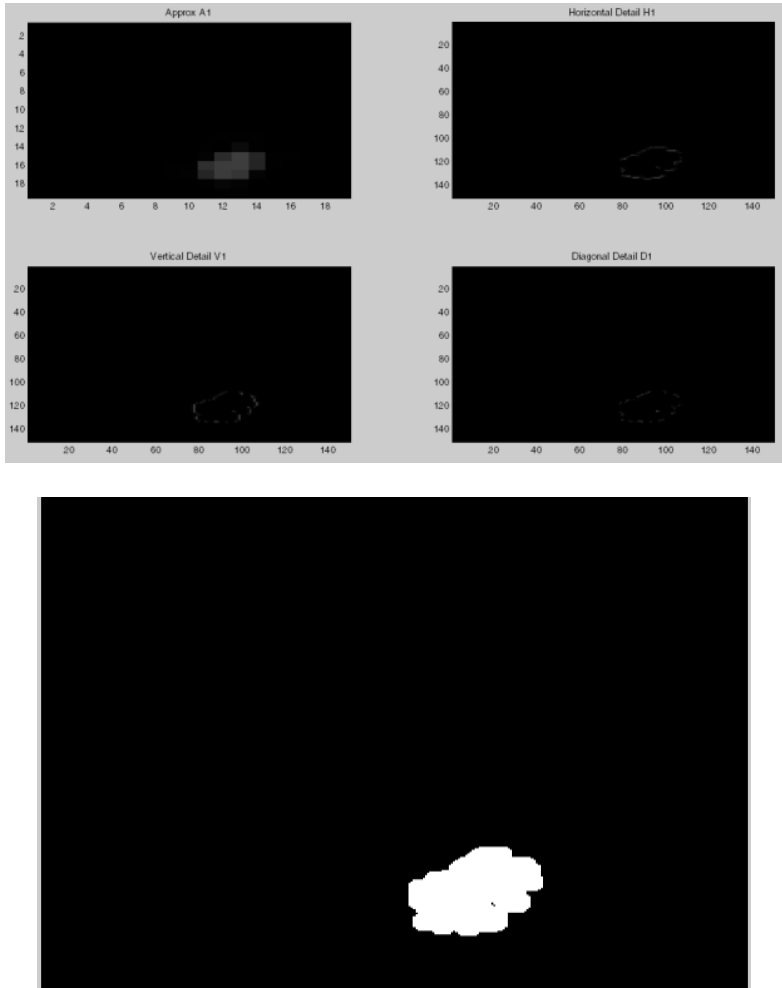

Fig.4. Morphological removed Image 

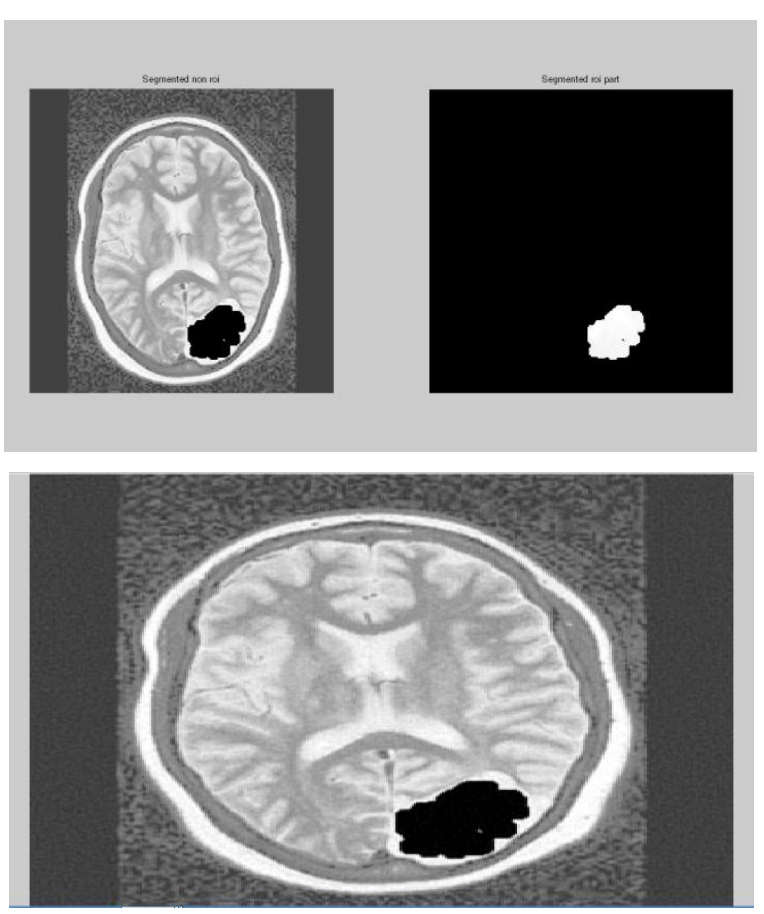

Fig.5. DCT compress image

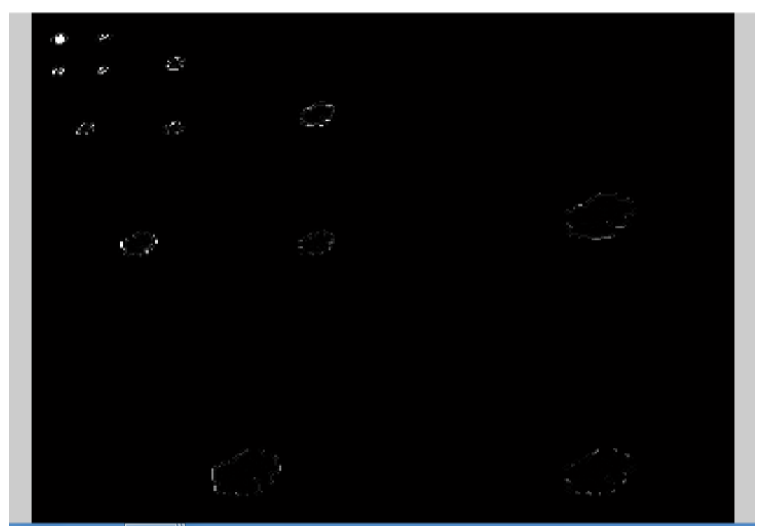

Fig.6. DWT Compress ROI image

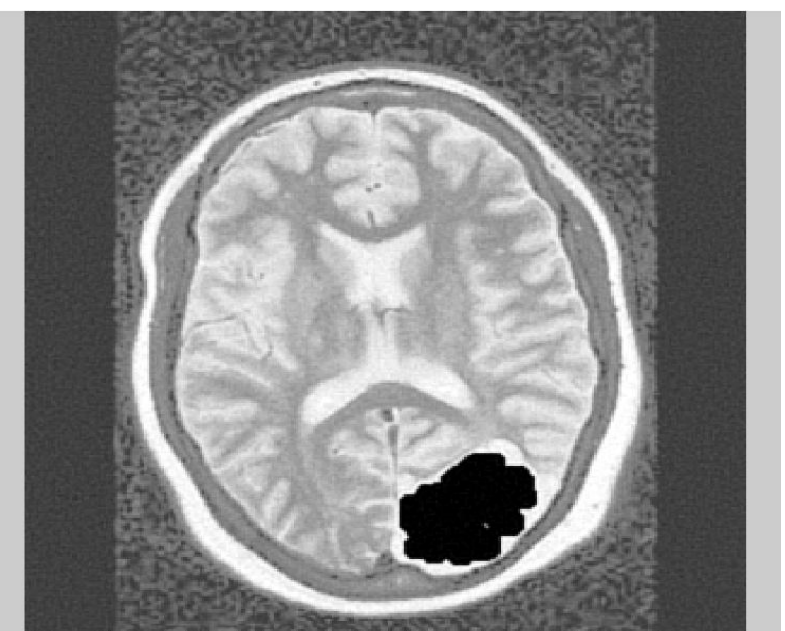

Fig.7. Reconstructed NON ROI image
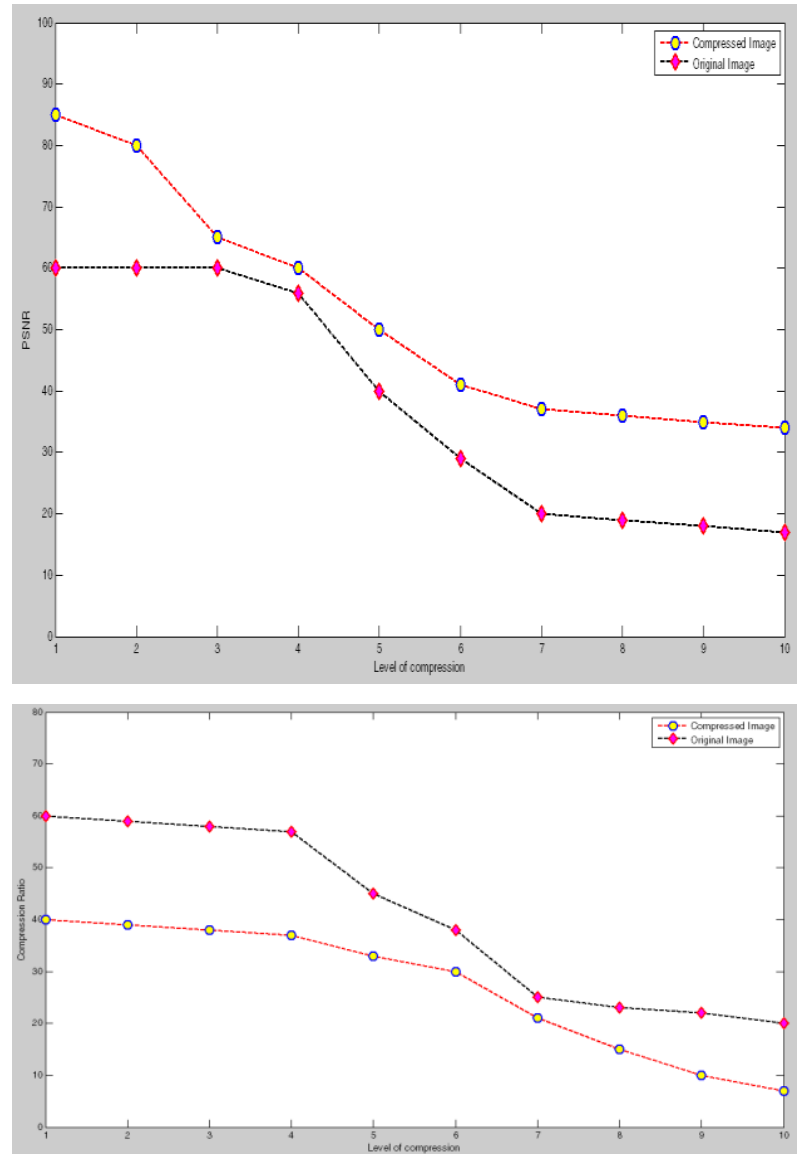

\section{CONCLUSION}

In this paper we have developed a procedure for line dependent wavelet transforms. We pointed out that this $s$ transform can be subjected to the encoder or the decoder and that it can hold compressed data. We provided an analysis for the condition in which both encoder and decoder are similar in terms of memory requirement and complexity. We explained highly scalable spilt coding algorithm that can work accompanied by a very low memory in set with the linedependent transform, and demonstrated that its behavior can be competitive accompanied by a state of the art image coders, at a fraction of their memory uses. To the best of our information, our work is the first to introduce a complete execution of a low memory wavelet image coder. Its another important advantage by creating a wavelet coder attractive both in terms of speed and memory requirements. This paper presents a novel wavelet-based approach for medical image fusion, which consists of 3 steps. In the 1th step, the medical images to be fused are decomposed into sub images by wavelet transform

\section{REFERENCES}

[1] Chandra Prakash, S Rajkumar and P.V.S.S.R. Chandra Mouli "Medical Image Fusion Based on Redundancy DWT and Mamdani Type Min-sum Mean-of-max Techniques with Quantitative Analysis" IEEE pp. 54-59 2012.

[2] Yong Yang, Dong Sun Park, Shuying Huang, and Nini Rao "Medical Image Fusion via an EffectiveWaveletBased Approach" EURASIP Journal on Advances in Signal Processing pp.1-13Volume 2010.

[3] Richa Singh, Mayank Vatsa and Afzel Noore "Multimodal Medical Image Fusion using Redundant 
DiscreteWavelet Transform".

[4] R.J.Sapkal, S.M.Kulkarni "Image Fusion based on Wavelet Transform for Medical Application" IJERAVol. 2, Issue 5, pp.624-627 Sep. - Oct. 2012.

[5] Yufeng ZHENG, E. A. ESSOCK, B. C. HANSEN, A. M. Haun "A new metric based on extended spatial frequency and its application to DWT based fusion algorithms"Elsevier pp.177-192 15 July 2005.

[6] J. Hajnal, D. G. Hill, and D. Hawkes. Medical Image Registration. CRC Press, 2001.

[7] Y. Kirankumar and S. Devi. Transform-based medical image fusion. International Journal of Biomedical Engineering and Technology, 1(1):101-110, 2007.

[8] Y. Licai, L. Xin, and Y. Yucui. Medical image fusion based on wavelet packet transform and self-adaptive operator. In Proceedings of International Conference on Bioinformatics and Biomedical Engineering, pages 2647-2650, 2008.

[9] D. Townsend and T. Beyer. A combined PET/CT scanner: the path to true image fusion. The British Journal of Radiology, 75:S24S30, 2002.

[10] Z. Wang and Y. Ma. Medical image fusion using $\mathrm{m}$ PCN. Information Fusion, 9(2):176-185, 2008.

[11] H. Zhang, L. Liu, and N. Lin. A novel wavelet medical image fusion method. In Proceedings of International Conference on Multimedia and Ubiquitous Engineering, pages 548-553, 2007. 